\title{
Ankle arthrodesis using computer assisted external fixator in patients with end-stage ankle arthrosis
}

\author{
Bulent Karslioglu $^{1 *}$, Ali Cagri Tekin ${ }^{1}$, Esra Tekin $^{2}$, Ersin Tasatan $^{1}$ \\ ${ }^{1}$ PROF. CEMIL TASCIOGLU CITY HOSPITAL, DEPARTMENTS OF ORTHOPEDICS AND TRAUMATOLOGY, ISTANBUL, TURKEY \\ ${ }^{2}$ PROF. CEMIL TASCIOGLU CITY HOSPITAL, DEPARTMENT OF ANESTHESIOLOGY, ISTANBUL, TURKEY
}

\section{ABSTRACT}

Objective. Ankle arthrosis is an end stage disease for the ankle that happens because of posttraumatic arthropathies or inflammatory diseases. Ankle arthrodesis is the gold standard for ankle arthritis. We present the functional outcome for a mid-term follow up study of the patients who underwent trans fibular ankle arthrodesis fixated with hexapod type external fixator through a distal fibular grafting technique. Materials and Methods. A number of 18 patients with trans fibular ankle arthrodesis were included in this study, out of which 6 were female and 12 were male. The mean age at surgery was 57.6 (ranging from 45 to 73). All the patients underwent follow up for a mean follow up period of 27.5 months (ranging from 24 to 35 months). One of the patients had undergone ankle-related surgery for 9 times. The others had undergone 4 or more ankle-related surgeries. Results. Ankle fusions resulted in bony union within postoperative 4.1 months (ranging from 3 to 6 months). None of our patients had superficial or deep infection, soft tissue problems or problems about the external fixator. The mean preoperative AOFAS score was 52.4, and the postoperative AOFAS score was 78.2. We had 7 excellent $(38.8 \%)$, and $11(61.1 \%)$ good results. We had two $\mathrm{cm}$ shortening in 2 patients, $1.5 \mathrm{~cm}$ shortening in 1 patient and $1 \mathrm{~cm}$ in 5 patients. Conclusions. We have found this method to be useful to be applied to patients, especially to those who undergo lots of surgeries and for whom amputation is a last option.

\section{Introduction}

Ankle arthrosis is an end-stage disease for the ankle joint that causes pain and ankle movement limitation. There are lots of causes for ankle arthritis. The most common reasons for this disease are inflammatory situations, chronic ankle instability such as Charcot arthropathies and related diseases, avascular necrosis of the talus and posttraumatic arthropathies [1].

There are currently two surgical options for ankle arthrosis: ankle arthrodesis (AA) and total ankle arthroplasty (TAA). AA was known to be the gold standard for end stage ankle arthritis (ESAA) [2].

Despite the improvement of the TAA implantation techniques and materials according to the current technological development, ankle arthrodesis is still the primary surgical option for ESAA because TAA is more surgeon and technique-dependent according to the AA, and TAA is still a strictly technically immature surgical option [3]. AA is also a salvage option for failed ankle arthroplasty.

AA is performed to eliminate painful ankle and to obtain a more functional and stable joint. There are lots of different techniques and fixation methods for AA. It can be done through open surgery or arthroscopically and fixations can be done with the help of screws, intramedullary nailing, plates or through external fixation.

In our study, we tried to explain our functional results of AA, which were fixated with computer assisted hexapod external fixator. We used distal fibula as a strut graft. There is no publication in the literature to report functional results over 2 years with computer-assisted ankle arthrodesis, as far as we know. 


\section{Materials and Methods}

A number of 18 patients with trans fibular ankle arthrodesis were included in this study. Out of them, 6 were female and 12 were male. The mean age at the time of surgery was 57.6 (ranging from 45 to 73 ). The mean follow-up period was 27.5 months (24-35 moths). A number of 5 patients had posttraumatic arthritis because of pilon fracture- one patient had severe osteomyelitis, 1 patient had congenital pesplanovalgus, 1 patient had sequala of pes equinovarus and 1 patient had posttraumatic arthritis after trimalleoler fracture (Table 1). A number 3 patients had diabetes mellitus, 2 patients had rheumatoid arthritis and 2 patients had osteoporosis, hypertension and congestive heart failure. One of the patients had undergone ankle-related surgery for 9 times. The others had undergone 4 or more ankle-related surgeries. All the participants were informed and the informed consent was obtained. The study was approved by our Institutional Review Board.

Table 1. Demographic characteristics and injury mechanisms of the patients

\begin{tabular}{|c|c|c|c|}
\hline \multicolumn{2}{|c|}{ Characteristics } & \multirow{2}{*}{$\begin{array}{c}\text { Total } \\
6\end{array}$} & \multirow{2}{*}{$\begin{array}{c}\% \\
33.3\end{array}$} \\
\hline Sex & Female & & \\
\hline & Male & 12 & 66.6 \\
\hline \multirow{4}{*}{$\begin{array}{c}\text { Injury } \\
\text { Mechanism }\end{array}$} & $\begin{array}{l}\text { Fracture (Pilon, } \\
\text { trimalleoler etc.) }\end{array}$ & 12 & 66.6 \\
\hline & $\begin{array}{l}\text { Pes equinovarus } \\
\text { sequel }\end{array}$ & 1 & 5.5 \\
\hline & Osteomyelitis & 3 & 16.6 \\
\hline & $\begin{array}{c}\text { Congenital } \\
\text { pesplanovalgus }\end{array}$ & 1 & 5.5 \\
\hline
\end{tabular}

Surgical Technique

An incision was made over the lateral aspect of the fibula beginning $10 \mathrm{~cm}$ proximal from the tip of the fibula with care being taken to avoid the injury to the sural nerve. Dissection is carried out to the basis of 4 metatarsals. The distal fibula exposed totally from soft tissues and osteotomy was performed at $6 \mathrm{~cm}$ proximal from the tip of the fibula. All ligamentous attachments of the fibula were resected, and the distal fibula was taken off entirely (Figure 1). The distal anterior part of the tibia dissected subperiosteally, the anterior capsule of the ankle joint was dissected, and so the distal tibia and the talus were visualized completely. Both articular cartilages removed with rongeur or burr and two smooth and no-cartilage covered surfaces were obtained. The hexapod type external fixator (HTEF) is applied in accordance with the surgical procedure. With slight decompression, all the surface of the ankle joint was visualized clearly and the surfaces in inaccessible areas were completely regulated. The excised distal fibula was peeled off from all cartilaginous and soft tissues. The ankle gap was measured, and four cortical sticks made according to the measurement. Two of the cortical sticks engrafted anteriorly and two other engrafted posteriorly. Cancellous grafts and remaining cortical chips grafts from the distal fibula were placed carefully to fill the entire ankle joint (Figure 2).

A spatial frame hexapod external fixator was established. The heel was placed in approximately 5degree valgus and the foot was placed in slightly external rotation compared to the opposite foot and the neutral flexion (Figure 3). Once the alignment was established, secure fixation was made with HTEF. Careful compression with HTEF was made to prevent shortening and to ensure the full contact of the arthrodesis surfaces. The alignment of the foot and the placement of the fixator was checked by means of fluoroscopy. Weight-bearing with modified shoe wearing was allowed immediately after surgery.

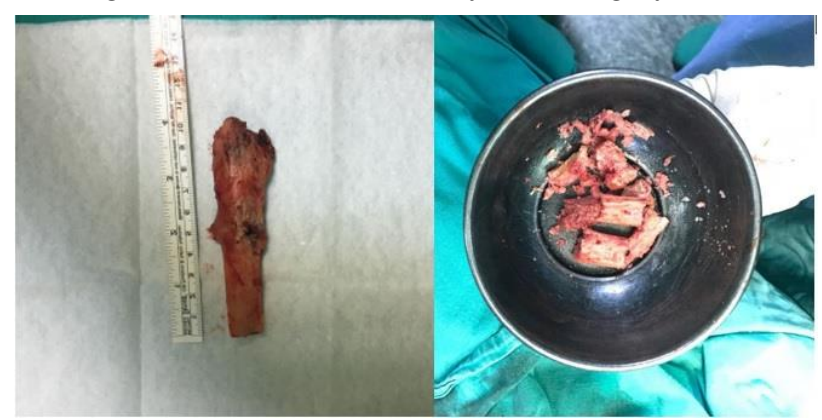

Figure 1. All ligamentous attachments of the fibula were resected and the distal fibula was taken off totally $6 \mathrm{~cm}$ proximal to the tip of the fibula

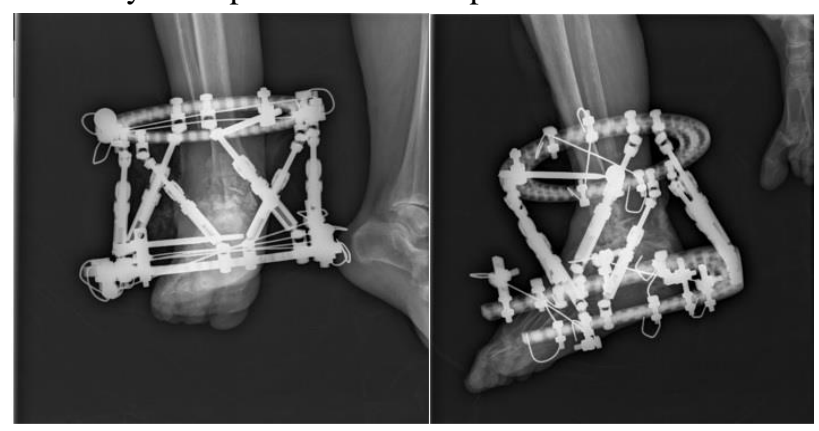

Figure 2. Cancellous grafts and remaining cortical chip grafts from the distal fibula were placed carefully to fill the entire ankle joint

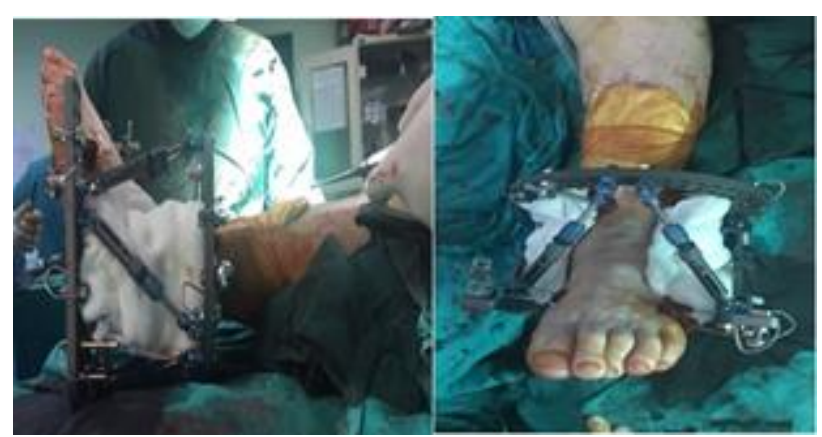

Figure 3. The heel was placed in approximately 5degree valgus and the foot was placed in slightly external rotation compared to the opposite foot and the neutral flexion 


\section{Results}

Ankle fusions resulted in solid bony union within 4.1 months postoperatively (ranging from 3 to 6 months) in all the patients. All the patients underwent follow-up radiologically and clinically for a mean follow-up of 27.5 months (ranging from 24 to 35 months). None of our patients had superficial or deep infection, soft tissue problems or problems about the external fixator.

All the patients were assessed with the American Orthopedic Foot and Ankle Society (AOFAS) AnkleHindfoot scale and with Visual analog score (Vas) according to their pain relief at the 6-month evaluation. The AOFAS score aims at evaluating pain, function and alignment of ankle-hindfoot. In our study, the mean preoperative AOFAS score was 52.4, and the postoperative AOFAS score was 78.2. We had 7 excellent (38.8\%), and $11(61.1 \%)$ good results. The preoperative mean VAS score was 3.1 (ranging from 2 to 4 ), the postoperative VAS score was 7.7 (ranging from 7 to 8) (Table 2). We had a two $\mathrm{cm}$ shortening in 2 patients, $1.5 \mathrm{~cm}$ shortening in 1 patient and $1 \mathrm{~cm}$ in the others. We detected a slight valgus deformity at the early postoperative period in 2 patients. Due to HTEF's flexible capability, we easily corrected the deformity. All the patients had a plantigrade foot and were happy because they had markedly reduced pain and they could manage their social lives on their own.

Table 2. The comparison of the patients' preoperative and postoperative AOFAS and Vas scores

\begin{tabular}{|l|c|c|}
\hline & Pre-op & Post-op (6 \\
\hline th & months $)$ \\
\hline VASAS & 52.4 & 78.2 \\
\hline
\end{tabular}

In our study, we had bone union in all patients, about 4.1 months after the surgery (Figure 4).

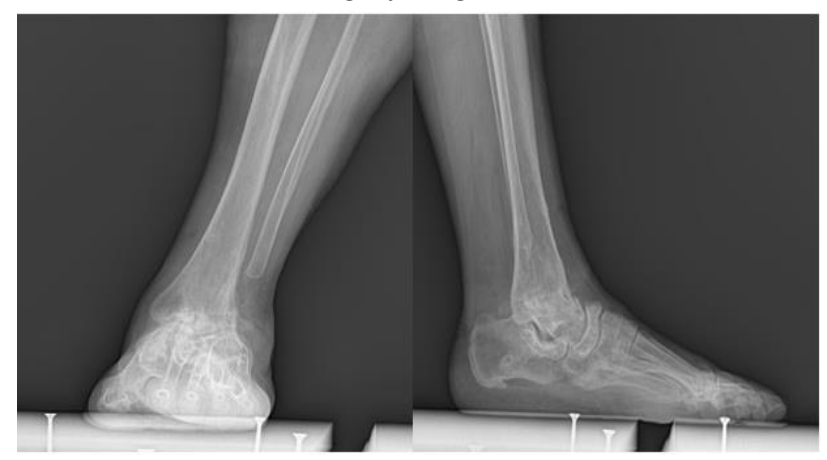

Figure 4. Postoperative 4-year control X-rays of one of our patients

\section{Discussion}

Painful ankle is a discomfort that severely affects the social life of patients. Feeling pain in every ankle's movement and having to use painkillers continuously is a devastating mood for a patient. Painful ankle may result from arthritis secondary to trauma, inflammatory situations, chronic ankle instability such as Charcot arthropathies and related diseases or avascular necrosis of the talus, etc. Ankle arthrosis is the natural end of painful ankle. Surgical interventions for ankle arthrosis are arthroscopic debridement, excision of impinging osteophytes and ankle arthrodesis (AA) or total ankle arthroplasty (TAA) for severe cases [2]. The goal of surgery is creating a pain free and plantigrade foot for healthy weight bearing.

Although AA remains the primary surgical treatment option for disabling ankle arthrosis [3], both AA and TAA are effective treatments for ankle arthrosis. AA is a definitive treatment for ankle arthrosis and, for that reason, patients typically have excellent pain relief according to TAA [4-6]. Although AA is highly superior in reducing pain, the ankle arc of motion is preserved in TAA. Nevertheless, reoperation and infection rates for the ankle joints are higher at TAA according to AA [7]. As a matter of fact, surgery of choice should be done by taking into consideration the patient's clinical and radiological characteristics.

The major complication of ankle arthrodesis is nonunion. The most important point of arthrodesis surgery is fulfilling the basics of fusion biomechanics: joint preparation, opposition and compression of joint surfaces in a desired arthrodesis position, as well as stable and firm fixation. If one or more of these rules are not fulfilled, nonunion is unavoidable.

Union rates at larger series range from $90 \%$ to $95 \%$ [8]. Preoperative and postoperative factors may lead to nonunion and, for that reason, avoidable factors should be determined carefully before surgery. Screening studies about this avoidable factor must focus on tobacco use, body mass index (BMI), Vitamin D abnormalities, endocrine disorders such as diabetes mellitus, thyroid dysfunction, parathyroid disease, calcium dysregulation, autoimmune disease, nutritional deficiencies, surgical site infection, vascular problems and the usage of some pharmaceutical agents such as high-dose corticosteroids and nonsteroidal anti-inflammatory medications $[9,10]$. Postoperatively, the most important factor is an inappropriate surgical technique. The inadequate preparation of the arthrodesis surface, thermal injury at the arthrodesis site or insufficient fixation method are surgery-dependent and avoidable factors. The accurate preoperative evaluation of the patient and meticulous surgery decreases the risk for nonunion. In our study, we had bony union approximately 4.1 months after surgery. We did not have patients with nonunion.

We performed fibular osteotomy and placed it as strut only graft to the arthrodesis site, which we included in our study. Autologous bone graft can be used to help achieving bone union if there is an infection, osteonecrosis of the 
talus, bone defects, as we had in our patients, and previous nonunion [3]. The iliac crest is an acceptable source for autologous bone graft, but it is associated with donor site morbidity [11]. The distal fibula can be used as a graft in different ways: fibular-only strut graft, as a lateral buttress [12], as slices of fibular bone graft [13], as intramedullary fibular strut graft [14], as vascularized pedicled graft [15], or as fibular strut only graft $[16,17]$. We peeled the excised distal fibula from all cartilaginous and soft tissues and prepared 4 cortical sticks. Two of the cortical sticks engrafted anteriorly and other two engrafted posteriorly. Cancellous grafts and remaining cortical chips grafts from distal fibula were placed carefully to fill the entire ankle joint. We achieved full bony union through this technique.

There are publications about two disadvantages of making fibular osteotomy. First, the fibula is the most important anatomic structure that supports the ankle joint laterally [16]. Fibular osteotomy weakens the lateral structures and leads to talus's lateral migration and so, the valgus deformity occurs at the ankle joint. We prevented this disadvantage by using HTEF permanently. HTEF made our work easier due to its three features. Firstly, if we needed more clear visualization of the ankle joint, we simply distracted the HTEF and compressed as much as we wanted during the surgery. Secondly, when the correct desired alignment of the foot was obtained, we used HTEF to lock-in joint at this position firmly and we could easily manipulate the joint. HTEF provides firm fixation in three planes against bending, torsional and shearing forces. Thirdly, we could directly see and adjust the disorders related to the alignment of the foot in the postoperative period.

The second disadvantage of fibular osteotomy is that it makes it difficult to determine the center of the rotation of the tibiotalar joint if we must convert ankle arthrodesis to ankle arthroplasty [16]. Determining the center of rotation of the tibiotalar joint plays an important role in TAA surgery and, for that reason, fibulectomy is a relative contraindication to the TAA [17]. Our selected patients had undergone ankle-related surgery more than 4 times and all of them had insufficient bone stock and severe osteoporosis in the ankle joint. We did not need to convert ankle arthrodesis to TAA because we applied this technique to well selected patients [18].

After ankle arthrodesis, the arc of motion through talonavicular and subtalar joints may increase. The firm fixation may help prevent subtalar arthrosis in particular. Adjacent joint disease in the future is an overlooked subject after arthrodesis surgery. It may be necessary to conduct a study on this topic in the future [19].

The small number of patients may be the weak point of our study, because we carefully evaluated the patients for surgical indications for this technique. Computer-aided external fixator systems have the disadvantage of being more expensive, besides the fact that there are advantages, such as allowing more interventions after surgery and obtaining better results with a good patient cooperation compared to the classical external fixator application.

\section{Conclusions}

Ankle fusions resulted in bony union within postoperative 4.1 months (ranging from 3 to 6 months) in all patients. None of our patients had superficial or deep infection, soft tissue problems or problems about the external fixator. We had 7 excellent $(38.8 \%)$, and 11 $(61.1 \%)$ good results, with two $\mathrm{cm}$ shortening in 2 patients, $1.5 \mathrm{~cm}$ shortening in 1 patient and $1 \mathrm{~cm}$ in 5 patients.

Therefore, we have found this method to be useful if applied to the patients who had undergone lots of surgeries and for whom amputation is a last option.

\section{Conflict of interest disclosure}

There are no known conflicts of interest in the publication of this article. The manuscript was read and approved by all authors.

\section{Compliance with ethical standards}

Any aspect of the work covered in this manuscript has been conducted with the ethical approval of all relevant bodies and that such approvals are acknowledged within the manuscript.

\section{Acronyms and abbreviations}

AA: Ankle arthrodesis

TAA: Total ankle arthroplasty

ESAA: End-stage ankle arthritis

HTEF: Hexapod type external fixator

AOFAS: American orthopedic Foot and Ankle Society

Vas: Visual analog score

BMI: $\quad$ Body mass index

\section{Author Contributions}

BK conceived the paper, oversaw the data collection, conducted the data analysis, wrote the manuscript. ACT participated in the study design, data analysis and interpretation, critically revised the manuscript and approved the final version. ET participated in the study design, data analysis and interpretation, critically revised the manuscript and approved the final version.

\section{References}

1. Pedersen E, Pinsker E, Younger AS, Penner MJ, Wing KJ, Dryden PJ, Glazebrook M, Daniels TR. Outcome of total ankle arthroplasty in patients with rheumatoid arthritis and noninflammatory arthritis. A multicenter 
cohort study comparing clinical outcome and safety. $J$ Bone Joint Surg Am. 2014 Nov 5;96(21):1768-75. doi: 10.2106/JBJS.M.01164

2. Mann RA, Van Manen JW, Wapner K, Martin J. Ankle fusion. Clin Orthop Relat Res. 1991 Jul;(268):49-55.

3. Nihal A, Gellman RE, Embil JM, Trepman E. Ankle arthrodesis. Foot Ankle Surg. 2008;14(1):1-10. doi: 10.1016/j.fas.2007.08.004

4. Morash J, Walton DM, Glazebrook M. Ankle Arthrodesis Versus Total Ankle Arthroplasty. Foot Ankle Clin. 2017 Jun;22(2):251-266. doi: 10.1016/j.fcl.2017.01.013

5. Gavrilă MT, Cristea S, Antoneac E. Study on the arthroscopic repair of the rotator cuff tear. J Clin Invest Surg. 2019;4(1):38-41. doi: 10.25083/2559.5555/4.1/38.41

6. Tudorachi NB, Eva I, Dascalu CG, AL-Hiary R, Barbieru B, Paunica M, Motofei C, Moraru AC. The influence of serum calcium and magnesium levels in the radiological evolution of knee osteoarthritis. $J$ Mind Med Sci. 2020;7(2):217-226. doi: 10.22543/7674.72.P217226

7. Younger AS, Glazebrook M, Veljkovic A, Goplen G, Daniels TR, Penner M, Wing KJ, Dryden PJ, Wong H, Lalonde KA. A Coding System for Reoperations Following Total Ankle Replacement and Ankle Arthrodesis. Foot Ankle Int. 2016 Nov;37(11):11571164. doi: 10.1177/1071100716659037

8. Chalayon O, Wang B, Blankenhorn B, Jackson JB 3rd, Beals T, Nickisch F, Saltzman CL. Factors Affecting the Outcomes of Uncomplicated Primary Open Ankle Arthrodesis. Foot Ankle Int. 2015 Oct;36(10):1170-9. doi: 10.1177/1071100715587045

9. Brinker MR, O'Connor DP, Monla YT, Earthman TP. Metabolic and endocrine abnormalities in patients with nonunions. J Orthop Trauma. 2007 Sep;21(8):557-70. doi: 10.1097/BOT.0b013e31814d4dc6

10. Moore KR, Howell MA, Saltrick KR, Catanzariti AR. Risk Factors Associated With Nonunion After Elective Foot and Ankle Reconstruction: A Case-Control Study. J Foot Ankle Surg. 2017 May-Jun;56(3):457-462. doi: 10.1053/j.jfas.2017.01.011
11. Banwart JC, Asher MA, Hassanein RS. Iliac crest bone graft harvest donor site morbidity. A statistical evaluation. Spine (Phila Pa 1976). 1995 May 1;20(9):1055-60. doi: 10.1097/00007632-19950500000012

12. Sung W, Greenhagen RM, Hobizal KB, Burns PR, Wukich DK. Technical guide: transfibular ankle arthrodesis with fibular-onlay strut graft. J Foot Ankle Surg. 2010;49(6):566-70. doi: 10.1053/j.jfas.2010.07.005

13. Ieong E, Mahapatra $P$, Nathan S. Fashioning autologous bone graft from the fibula in the transfibular approach to open ankle arthrodesis. Foot Ankle Surg. 2014 Jun;20(2):149-50. doi: 10.1016/j.fas.2013.12.002

14. Shah AB, Jones C, Elattar O, Naranje SM. Tibiotalocalcaneal Arthrodesis With Intramedullary Fibular Strut Graft With Adjuvant Hardware Fixation. J Foot Ankle Surg. 2017 May-Jun;56(3):692-696. doi: 10.1053/j.jfas.2017.01.055

15. Roukis TS, Kang RB. Vascularized Pedicled Fibula Onlay Bone Graft Augmentation for Complicated Tibiotalocalcaneal Arthrodesis With Retrograde Intramedullary Nail Fixation: A Case Series. J Foot Ankle Surg. 2016 Jul-Aug;55(4):857-67. doi: 10.1053/j.jfas.2015.12.003

16. Coskun HS, Mirac Baris Usta MB, Say F, Yildirim AM. Factors affecting post-traumatic stress disorder in children with orthopedic injury. J Clin Invest Surg. 2020; 5(1): 24-28. doi: 10.25083/2559.5555/5.1/24.28

17. Miulescu RD, Guja L, Ochiana LC, Ungurianu A, Șeremet OC, Ștefănescu E. Serum markers of bone fragility in type-2 diabetes mellitus. J Mind Med Sci. 2019; 6(1): 78-85. doi: 10.22543/7674.61.P7885

18. Firoozabadi R, Thuillier D, Benirschke S. Obtaining Correct Ankle Alignment Using Intraoperative External Fixation for Ankle Arthrodesis. J Foot Ankle Surg. 2017 Mar-Apr;56(2):242-246. doi: 10.1053/j.jfas.2016.11.016

19. Hintermann B, Barg A, Knupp M, Valderrabano V. Conversion of painful ankle arthrodesis to total ankle arthroplasty. J Bone Joint Surg Am. 2009 Apr;91(4): 850-8. doi: 10.2106/JBJS.H.00229 